\title{
Aspects of $\mathrm{N}=1$ String Dynamics *
}

\author{
Shamit Kachru ${ }^{\mathrm{a}}$

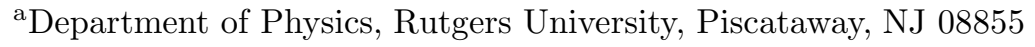

\begin{abstract}
We review several topics of interest in the study of $4 \mathrm{~d} N=1$ supersymmetric compactifications of the heterotic string. After a brief introduction to the construction of such models, our focus is on the novel physics which occurs at singularities in the moduli space of vacua. Among the phenomena we discuss are nonperturbative superpotentials, dynamical generation of poles in various low-energy couplings, and phase transitions which change the net number of chiral generations.
\end{abstract}

\section{Introduction}

Much has been learned from string duality about superstring vacua with extended supersymmetry (for reviews of various aspects, see e.g. (1]). For instance:

- Naively distinct moduli spaces are smoothly connected (e.g. through conifold transitions in $4 \mathrm{~d}$ $\mathrm{N}=2$ type II vacua (2, 4)

- Dual descriptions allow one to compute nonperturbative effects in one picture by doing treelevel computations in a dual theory (e.g. in heterotic/type II duality in four dimensions with $\mathrm{N}=2$ supersymmetry [5.6])

While we now have an excellent understanding of most phenomena which occur in string vacua with extended supersymmetry, our understanding of $4 \mathrm{~d} N=1$ vacua is much less complete. There are several motivations for studying such vacua:

- Less supersymmetry allows richer phenomena. For example, in $\mathrm{N}=1$ theories the classical moduli space can be lifted by quantum effects, i.e. supersymmetry can be dynamically broken. This is impossible in theories with extended supersymmetry.

- $4 \mathrm{~d} \mathrm{~N}=1$ is the case which might be relevant to the real world. $\mathrm{N}=1$ allows chiral gauge representations, while for extended supersymmetric theories one typically has only nonchiral representations. In addition, weak scale SUSY is one plausible explanation for the unnaturally large ratio of the weak scale to the Planck scale, the so-called

\footnotetext{
*Lectures presented at the 33rd Karpacz Winter School "Duality: Strings and Fields" (Poland, February 1997).
}

"hierarchy problem." Finally, the observed unification of the gauge couplings in minimal supersymmetric extensions of the standard model leads one to suspect that $\mathrm{N}=1$ SUSY may be lurking around the corner at the weak scale.

- $N=1$ SUSY is much more "generic" than extended SUSY, just in terms of number of vacua. Such vacua should be studied for this reason alone.

\section{2. $\mathrm{N}=1$ Theories from String Compactifi- cation}

There are several different ways to get $4 \mathrm{~d} N=1$ theories out of string theory and its parent theories, M-theory and F-theory. We describe a few methods of constructing $\mathrm{N}=1$ theories below. For most of these lectures, our focus will be on the heterotic $S O(32)$ and $E_{8} \times E_{8}$ strings.

\subsection{Heterotic Theories}

One can start with either $E_{8} \times E_{8}$ or $S O(32)$ gauge group in ten dimensions. Then the low energy effective theory is 10d N=1 Super YangMills, coupled to supergravity. If we look for solutions of this theory preserving one fourdimensional supersymmetry, we find [7]:

- The compactification space $M$ must be a manifold of $S U(3)$ holonomy, a Calabi-Yau manifold. The reduced holonomy implies that the first Chern class $c_{1}(M)$ vanishes. Such manifolds are complex Kahler manifolds and can be equipped with a Ricci-flat metric for each choice of their complex structure and Kahler class $J$. The parameters involved in these choices yield scalar 
moduli in the $4 \mathrm{~d} \mathrm{~N}=1$ effective field theory. One obtains $h^{2,1}(M)$ such moduli parametrizing the choice of complex structure, and $h^{1,1}(M)$ giving the choice of Kahler class $J$.

- The gauge field strength $F$ must satsify the equations

$$
F_{i j}=F_{\overline{i j}}=0, \quad g^{i \bar{j}} F_{i \bar{j}}=0
$$

where $i$ and $\bar{i}$ label (anti)holomorphic coordinates on the complex manifold $M$ and $g$ is the Ricciflat Kahler metric on $M$. These equations have a solution iff the gauge connection is a connection on a stable, holomorphic vector bundle $V \rightarrow M$ [8]. Furthermore, there is an integrability condition for the solution of the Kahler Yang-Mills equations

$$
\int_{M} J \wedge J \wedge c_{1}(V)=0
$$

Also, the Bianchi identity for the three-form field strength $H$ requires

$d H=\operatorname{Tr}(R \wedge R)-\operatorname{Tr}(F \wedge F) \rightarrow c_{2}(T M)=c_{2}(V)$

Typically, the vector bundles $V$ come in multiparameter families parametrized by elements of $H^{1}(M, \operatorname{End}(V))$.

\section{EXAMPLES}

There are relatively simple techniques for constructing many examples of Calabi-Yau threefolds; for a deeper understanding, one should consult e.g. [9]. The simplest examples involve constructing the Calabi-Yau as a hypersurface or a complete intersection of hypersurfaces in some weighted projective space.

The projective space $C P^{N}$ is simply a space defined by homogeneous complex coordinates $z_{1}, \cdots, z_{N+1}$ subject to the relation

$$
\left(z_{1}, \cdots, z_{N+1}\right) \sim\left(\lambda z_{1}, \cdots, \lambda z_{N+1}\right)
$$

for any nonzero complex $\lambda$, and with the origin removed. Using the adjunction formula [9], it is easy to see that a hypersurface $M$ defined by an equation which is homogeneous of degree $N+1$ in the $z_{i}$ will have $c_{1}(M)=0$. To get a threefold in this way, one needs to work in $C P^{4}$, and one obtains the famous quintic in $C P^{4}$. Similarly, any intersection of $k$ hypersurfaces, with defining polynomials whose degrees sum to $N+1$, will define a Calabi-Yau $N-k$ fold in $C P^{N}$.

A simple generalization of the complex projective space $C P^{N}$ is the weighted projective space $W C P_{w_{1}, \cdots, w_{N+1}}^{N}$ defined by homogeneous coordinates $z_{1}, \cdots, z_{N+1}$ subject to an identification

$$
\left(z_{1}, \cdots, z_{N+1}\right) \sim\left(\lambda^{w_{1}} z_{1}, \cdots, \lambda^{w_{N+1}} z_{N+1}\right)
$$

for any nonzero complex $\lambda$ (and again with the origin removed). One calls a polynomial $F\left(z_{i}\right)$ homogeneous of degree $d$ in this weighted projective space if

$$
F\left(\lambda^{w_{1}} z_{1}, \cdots, \lambda^{w_{N+1}} z_{N+1}\right) \sim \lambda^{d} F\left(z_{1}, \cdots, z_{N+1}\right)
$$

It again follows from the adjunction formula that a complete intersection of $k$ hypersurfaces whose degrees add up to $\sum w_{i}$ will define a Calabi-Yau $N-k$ fold in the $W C P^{N}$. There are subtleties in certain cases, involving singularities of the ambient $W C P^{N}$ which might be inherited by the Calabi-Yau; we will not discuss these issues here, and refer the reader to [9].

We have not mentioned the construction of appropriate gauge bundles here. Each Calabi-Yau comes equipped with its tangent bundle, which serves as a suitable choice. However, one should keep in mind that this choice, "embedding the spin connection in the gauge group," gives only a very small class of vacua. More general choices will be briefly discussed below in $\S 3.1$.

There are two important points to emphasize about our whole discussion of heterotic vacua:

i) The conditions we've discussed above are sufficient to guarantee a solution to all orders in the string worldsheet sigma model coupling $\alpha^{\prime}$. Nonperturbatively in $\alpha^{\prime}$ we must also worry about so-called "worldsheet instanton" effects [10]. In certain classes of examples, it is known that such effects do not spoil conformal invariance [11].

ii) The heterotic $S O(32)$ and type I theories both have 10d N=1 Super-Yang Mills coupled to $S O(32)$ gauge fields as their low energy limits. They are in fact identical theories, equivalent under the strong/weak duality transformation 12]

$$
g_{M N}^{h e t}=\lambda^{h e t} g_{M N}^{I}, \quad \lambda^{h e t}=\frac{1}{\lambda^{I}}
$$


where $\lambda$ denotes the string couplings. In particular, this implies that after compactification on a Calabi-Yau threefold $M$,

$$
\left(e^{\frac{-R^{2}}{\alpha^{\prime}}}\right)_{h e t} \rightarrow\left(e^{\frac{-R^{2}}{\alpha^{\prime} \lambda_{I}}}\right)_{I}
$$

Here, $R^{2}$ is the size of some rational curve in $M$. So heterotic worldsheet instanton effects map to type I spacetime instanton effects! One should think of the heterotic string as the Dirichlet 1brane of the type I theory, so in particular the 1 brane wrapping a holomorphic curve in a D5 brane is a "spacetime" instanton effect in the D5 brane worldvolume quantum field theory [13.

\subsection{M-theory}

One can get $4 \mathrm{~d} N=1$ theories from M-theory by compactifying on a 7 -manifold of $G_{2}$ holonomy. Examples of such theories have been studied [14] but not much is known about their physics.

\subsection{F-theory}

One gets a $4 \mathrm{~d} \mathrm{~N}=1$ theory by compactifying F-theory [15] on a Calabi-Yau fourfold. We have two comments to make about such theories:

i) The type IIA string has an interaction [16]

$$
\delta S=-\int B \wedge X_{8}(R)
$$

where B is the NS-NS 2-form and $X_{8}$ is a quartic polynomial in the curvature. So if one compactifies on a fourfold $X$ with

$$
I=-\int_{X} X_{8}(R) \neq 0
$$

then naively there is a vacuum destabilizing tadpole for the $B$ field. This lifts to a similar term in F-theory involving the 4-form gauge field which couples to the $\mathrm{SL}(2, \mathrm{Z})$ invariant three-brane. One can show that

$$
I=\frac{\chi}{24}
$$

for $X$ a Calabi-Yau. In order to cancel the tadpole, one can include $I$ threebranes localized on $X$ but filling the transverse spacetime $R^{3,1}$ 17. .

\footnotetext{
${ }^{2}$ Note that if $I$ is negative, there is no way to cancel the tadpole and preserve supersymmetry.
}

ii) It follows from the adiabatic argument and the duality between the heterotic string on $T^{2}$ and Ftheory on elliptic $K 3$ surfaces that there are also dualities between $4 \mathrm{~d} N=1$ heterotic and F-theory compactifications. Suppose $X$ is an elliptic fourfold with base $B$, and $B$ is fibered over $B^{\prime}$ with $P^{1}$ fibers. Then the adiabatic argument suggests that F-theory on $X$ is dual to the heterotic string on the elliptic Calabi-Yau threefold $Y$ with base $B^{\prime}$. The $I$ three-branes present in the F-theory compactification map, in the heterotic theory, to $I$ 5-branes wrapped on the elliptic fiber of $Y$.

\section{More About Classical $\mathrm{N}=1$ Heterotic Theories}

For most of these lectures, our focus will be on understanding heterotic compactifications on Calabi-Yau threefolds. The first step is to obtain a proper understanding of the classical $\mathrm{N}=1$ heterotic vacua. We discussed the classical geometry briefly in $\S 2.1$, but in string theory the classical vacua are really worldsheet conformal field theories. These can predict interesting "quantum geometrical" phenomena when some length scales in the compactification geometry shrink to the string length.

The worldsheet data required to specify a "realistic" $S O(32)$ heterotic string vacuum of the form $M_{4} \times M$ where $M_{4}$ is Minkowski space and $M$ is some compact manifold is:

- A free theory with left and right central charges $c_{L}=4, c_{R}=6$ for the $M_{4}$ CFT.

- A $c_{L}=16-N$ left-moving CFT composed of free fermions, representing the $S O(32-2 N)$ current algebra.

- A $c_{L}=6+N, c_{R}=9$ conformal theory representing the "internal" Calabi-Yau sigma model with $(0,2)$ supersymmetry. The left moving fermions couple to an $S U(N)$ bundle $V \rightarrow M$.

These are the basic structures we need. To see if the classical string theory differs in any interesting way from the classical geometry, we need a considerably more precise description of the Calabi-Yau piece of the conformal field theory. 


\subsection{Gauged Linear Sigma Models}

It is prohibitively difficult in most cases to directly construct the $2 \mathrm{~d}$ quantum field theory as an exact conformal field theory. What one can do, following Witten [18], is construct a $(0,2)$ gauged linear sigma model that should be taken under RG flow to the desired $(0,2)$ conformal field theory in the infrared. The parameters in this gauged linear sigma model then represent the spacetime moduli of the string theory.

We will now try to construct a $(0,2)$ supersymmetric QFT that will flow, in some limit, to a sigma model describing strings propagating on a complete intersection Calabi-Yau in a weighted projective space. The relevant $(0,2)$ superspace has supercharges $Q^{+}$and $\bar{Q}^{+}$and SUSY multiplets:

$$
\begin{gathered}
\text { Chiral : } \Phi=\phi+\theta^{+} \psi+\theta^{+} \bar{\theta}^{+} \partial_{\bar{z}} \phi \\
\text { Fermi : } \Lambda=\lambda+\theta^{+} l+\theta^{+} \bar{\theta}^{+} \partial_{\bar{z}} \lambda
\end{gathered}
$$

where $z, \bar{z}$ are complex worldsheet coordinates and the $\theta$ s are the fermionic superspace coordinates. In such a superspace, we consider a $U(1)$ gauge theory the following field content. We take $Q+4$ chiral multiplets $\Phi^{i}$ with gauge charges $w_{i}$ and one additional chiral multiplet $P$ with charge $-m$. We also take $N+1$ fermi multiplets $\Lambda^{a}$ with charges $n_{a}$ and $Q$ fermi multiplets $\Sigma_{j}$ with charges $-d_{j}$. We will see momentarily that there are constraints on the various charges, following from the conditions discussed in $\$ 2.1$.

In addition to normal kinetic terms for all of the fields, we will include two special terms in the action:

1) A Fayet-Iliopoulos D-term for the U(1) gauge field

$$
S_{D}=r \int d^{2} z D-i \frac{\theta}{2 \pi} \int d^{2} z f
$$

where $D$ is an auxiliary field in the $\mathrm{U}(1)$ gauge multiplet and $f$ is the $\mathrm{U}(1)$ field strength.

2) A $(0,2)$ superpotential

$$
S_{W}=\int d^{2} z d \theta^{+} \Sigma_{j} W^{j}(\Phi)+P \Lambda^{a} F_{a}(\Phi)
$$

where $\mathrm{U}(1)$ gauge invariance dictates that $W^{j}$ should be a polynomial in the $\Phi_{\mathrm{s}}$ with charge $d_{j}$ and $F_{a}$ should have charge $m-n_{a}$.
With this Lagrangian, integrating out auxiliary fields leads to a potential energy

$$
\begin{gathered}
U(\phi)=\sum_{j=1}^{Q}\left|W_{j}(\phi)\right|^{2}+|p|^{2} \sum_{a}\left|F_{a}(\phi)\right|^{2} \\
+\frac{e^{2}}{2}\left(\sum_{i=1}^{Q+4} w_{i}\left|\phi_{i}\right|^{2}-m|p|^{2}-r\right)^{2}
\end{gathered}
$$

The infrared behavior of the quantum field theory will be governed by the vacua of $U(\phi)$, at least for large $|r|$ when the excitations around the minima are very massive. There are two such regimes:

\section{(I) The Calabi-Yau Phase}

Take $r>>0$. Assume $F_{a}=W_{j}=0 \rightarrow \phi_{i}=0$; we will explain this momentarily. Then the locus of $U(\phi)=0$ is

$$
p=0, \quad \sum_{i} w_{i}\left|\phi_{i}\right|^{2}=r, \quad W_{j}(\phi)=0
$$

Dividing

$$
\sum_{i} w_{i}\left|\phi_{i}\right|^{2}=r
$$

by the $U(1)$ symmetry leaves a copy of the $Q+3$ dimensional weighted complex projective space with weights $w_{1}, \cdots, w_{Q+4}$, and with Kahler class (or "size") $r$. Then $W_{j}(\phi)=0$ defines a complete intersection Calabi-Yau manifold $M$ in this weighted projective space, as long as we require $\sum d_{j}=\sum w_{i}$.

Further examination of the Yukawa couplings of the worldsheet fermions, as in 19], reveals that the massless combinations of the right-movers $\psi_{i}$ transform as sections of $T M$, while the leftmovers transform as sections of a holomorphic bundle $V \rightarrow M$, with holomorphic structure defined by the $F_{a}(\phi)$. More precisely, this bundle is defined as the kernel of an exact sequence

$$
0 \rightarrow V \rightarrow \bigoplus_{a=1}^{N+1} \mathcal{O}\left(n_{a}\right) \stackrel{\otimes F_{a}(\phi)}{\longrightarrow} \mathcal{O}(m) \rightarrow 0
$$

We now recognize that

$$
W_{j}(\phi)=F_{a}(\phi)=0 \rightarrow \phi_{i}=0
$$


is necessary simply for the geometry of $V \rightarrow M$ to be smooth. Requiring that $V$ satisfy the constraints of $\S 2.1$ then constrains the $U(1)$ gauge charges of various worldsheet multiplets:

$$
\begin{gathered}
c_{1}(V)=0 \rightarrow m=\sum n_{a} \\
c_{2}(V)=c_{2}(T M) \rightarrow \\
m^{2}-\sum n_{a}^{2}=\sum d_{j}^{2}-\sum w_{i}^{2}
\end{gathered}
$$

In fact, both conditions are simply anomaly cancellation conditions on the worldsheet 18, 19. The first requires that there be a non-anomalous $\mathrm{U}(1)$ global symmetry acting on the left movers, while the second is simply the condition that the worldsheet U(1) gauge anomaly should vanish!

In this framework, the spacetime moduli fields appear as follows:

- Changes of $(r, \theta)$ correspond to varying the (complexified) Kahler moduli

- Polynomial deformations of $W_{j}(\phi)$ correspond to moduli of the complex structure of $M$

- Polynomial deformations of $F_{a}(\phi)$ correspond to moduli of the holomorphic bundle $V$

So for $r>0$ we recovered the CalabiYau nonlinear sigma model, with the parameters (moduli) we expect of the $(0,2)$ CFT. This limit reproduces what we expect from classical geometry. What about the other phase, with $|r|>>0$ but $r<<0$, which can also be studied semiclassically?

\section{(II) The Landau-Ginzburg Phase}

For $r<<0$, the minimum of $U(\phi)$ occurs when $|p|^{2}=-\frac{r}{m}$ and $\phi_{i}=0$. The $\mathrm{U}(1)$ gauge symmetry is broken to a $Z_{m}$ by $\langle p\rangle$, which carries nonzero $\mathrm{U}(1)$ charge. The massless degrees of freedom are the $\phi \mathrm{s}, \Sigma \mathrm{s}$, and $\Lambda \mathrm{s}$, governed by a superpotential

$$
W=\Sigma^{j} W_{j}(\phi)+\Lambda^{a} F_{a}(\phi)
$$

The unbroken $Z_{m}$ discrete gauge symmetry instructs us to take a $Z_{m}$ orbifold of the naive Landau-Ginzburg theory, to obtain a so-called "Landau-Ginzburg orbifold."

\footnotetext{
${ }^{3}$ In fact, as long as the $F_{a}(\phi)$ are sufficiently generic, mild singularities of $V$ or rather drastic singularities of $M$ may be present without adversely affecting the perturbative string theory.
}

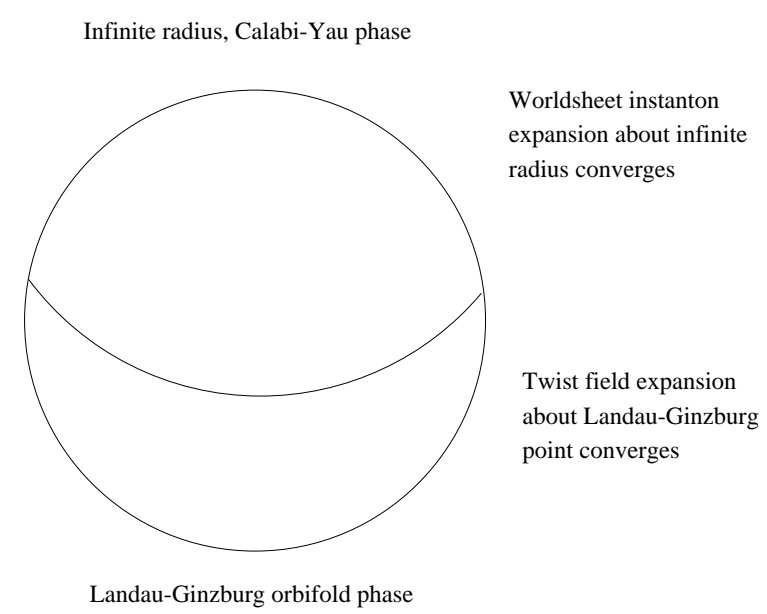

FIGURE 1: A picture of the Kahler moduli space.

So the global picture of the Kahler moduli space, controlled by $(r, \theta)$, is roughly as follows. As $r$ decreases from $\infty$ to 0 , the Calabi-Yau sigma model coupling gets stronger and stronger until finally the perturbation series for various correlators diverges. Going "past" this point, to negative $r$, one finds that there is a new convergent perturbation expansion, this time in twist fields about the Landau-Ginzburg orbifold, which becomes more and more weakly coupled as $r \rightarrow$ $-\infty$. This is summarized in Figure 1 above.

\subsection{A Glimpse of Quantum Geometry}

The Landau-Ginzburg phases are as "far away" from the $r \rightarrow \infty$ classical geometry limit as we can get in the Calabi-Yau moduli space. One might therefore expect that examples of "stringy" quantum geometrical effects might be particularly manifest there. One such phenomenon, which occurs in string theory but not classical geometry and is mediated through the LandauGinzburg phase, is a certain kind of smooth topology change [19 21].

Notice at the Landau-Ginzburg point, there is no invariant distinction between the $\Sigma_{j}$ s and the $\Lambda^{a} \mathrm{~s}$ (or the $W_{j} \mathrm{~s}$ and the $F_{a} \mathrm{~s}$ ). This is in marked distinction to the distinguished roles these fields play at large radius, where the $W_{j}$ s determine $M$ and the $F_{a}$ s determine the holomorphic structure 
of $V$. This makes it possible for us to imagine an automorphism which exchanges certain $\Sigma$ s and $\Lambda \mathrm{s}$, while at the same time exchanging certain $W \mathrm{~s}$ and $F$ s, at the Landau-Ginzburg point. Since the spectrum of chiral fields remains invariant under such a re-labeling, any models related by such a symmetry must (at large radius) be Calabi-Yau complete intersections in the same weighted projective space. Examples of such automorphisms yield pairs of topologically distinct Calabi-Yau manifolds $M, X$ (which can even have different Euler characters $\chi(M) \neq \chi(X))$ and vector bundles $V \rightarrow M$ and $E \rightarrow X$ which are smoothly connected through their (shared) Landau-Ginzburg phase. I The Landau-Ginzburg phase then plays the role of a multi-critical point in the CFT moduli space. One can turn on distinct moduli, which are not mutually integrable, to go to large-radius in either of the two distinct Calabi-Yau phases. Simple concrete examples of this phenomenon are discussed in [19 21] and we will give no further details here.

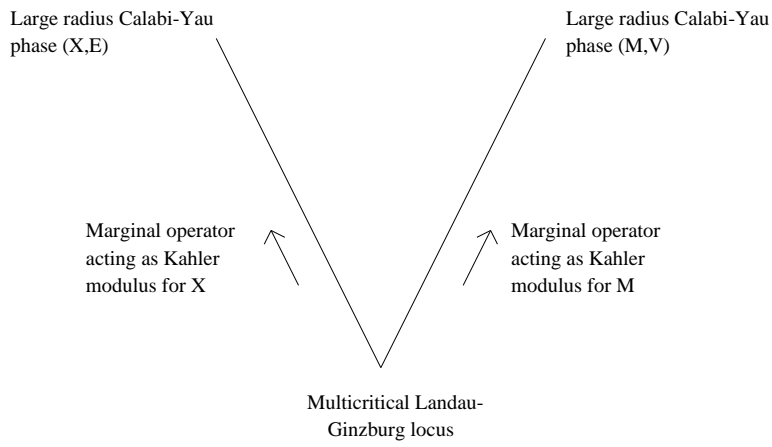

FIGURE 2: Topology change through LG phase.

\section{General Remarks about Singularities}

As discussed by many other lecturers, SUSY field theories and string theories typically have moduli spaces $\mathcal{M}$ of physically inequivalent vacua parametrized by scalar VEVs $\langle\phi\rangle$. These moduli

\footnotetext{
$\overline{{ }^{4} \text { In such examples, } \int_{M}} c_{3}(V)=\int_{X} c_{3}(E)$; the net space-
} time chirality does not change. spaces $\mathcal{M}$ typically have special points $m^{*} \in \mathcal{M}$ where the physics is singular. Interesting dynamics occurs there. A lesson of the recent advances is that understanding what happens at singular points in the passage from the classical to the quantum theory is the crucial step in "solving" the quantum theory.

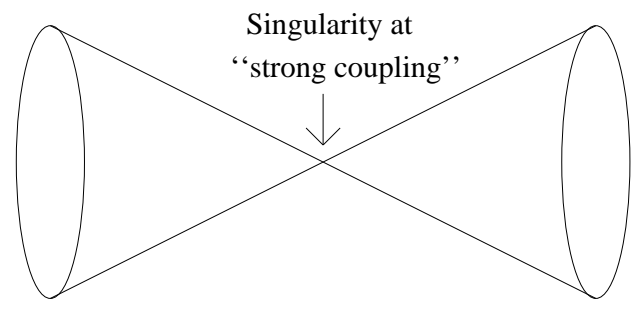

$|\phi|>>\Lambda$

Another weakly semiclassical regime coupled regime

FIGURE 3: A depiction of some "typical" moduli space in a theory with scale $\Lambda$.

\subsection{Some Examples}

Let us begin by reviewing some prototypical examples. At least four sorts of phenomena have been observed in $4 \mathrm{~d}$ gauge theory and string theory:

1) A classical singularity is removed in the quantum theory.

Example: Consider the $S U(2)$ gauge theory with $N_{F}=2$ flavours of quarks, i.e. four doublets $d_{i}$, $1 \leq i \leq 4$. The coordinates on the classical moduli space $\mathcal{M}_{c l}$ are $V_{i j}=\epsilon_{\alpha \beta} d_{i}^{\alpha} d_{j}^{\beta}$. The equation defining $\mathcal{M}_{c l}$ is

$$
\mathcal{M}_{c l}: \operatorname{Pf}(V)=\epsilon^{i j k l} V_{i j} V_{k l}=0
$$

In the quantum theory, this is deformed to

$$
\mathcal{M}_{q u}: \operatorname{Pf}(V)=\Lambda^{4}
$$

where $\Lambda$ is the scale of the gauge theory. The singular point $V_{i j}=0$ on the classical moduli space, where the $S U(2)$ gauge symmetry is restored, is removed in the quantum theory [23].

2) The mathematical structure of $\mathcal{M}_{c l}$ and $\mathcal{M}_{q u}$ agrees, but the physical interpretation of the singular points changes. 
Example: Consider $S U\left(N_{c}\right)$ gauge theory with $N_{F}=N_{C}+1$ flavours of quarks. Then the classical singular points are due to the appearance of massless gluons ("unHiggsing"), while the quantum singularities are due to the appearance of additional massless composite "baryons" and "mesons" 23].

3) There are still singularities on $\mathcal{M}_{q u}$, but the mathematical structure (and hence also the physical interpretation) is modified.

Example: Take the famous case of $\mathrm{N}=2$ supersymmetric pure $S U(2)$ gauge theory. The $\mathrm{N}=2$ gauge multiplet contains a complex scalar $\phi$ in the adjoint of $S U(2)$. There is a one complex dimensional moduli space of vacua, parametrized by the gauge invariant coordinate $u=\operatorname{Tr}\left(\phi^{2}\right)$. In the classical moduli space, there is a singular point at $u=0$ where the $S U(2)$ gauge symmetry is restored. In the quantum theory, this splits into two singular points at $u= \pm \Lambda^{2}$ (where $\Lambda$ is the scale of the theory), where a monopole and a dyon become massless. This is the crucial insight needed to solve the theory [24.

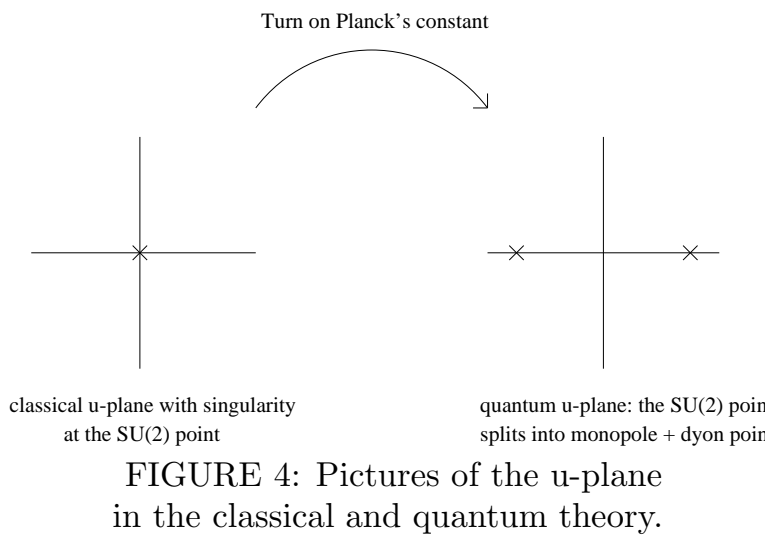

There is a fourth possibility which seems to occur in string theory. Namely:

4) The singularity is 'inexplicable' classically. In the quantum theory, it arises due to quantum loops or nonperturbative dynamics of solitons. These (quantum) effects are independent of $g_{\text {string }}$ because of the unusual couplings of these solitons.

Example: A famous example is provided by Strominger's resolution of conifold singularities in IIB compactifications on Calabi-Yau threefolds [3]. There, $M$ is a Calabi-Yau and $X \subset M$ a three-cycle. There is a scalar component $z$ in a Ramond-Ramond $U(1)$ vector multiplet given by

$$
z=\int_{X} \Omega
$$

One can wrap a Dirichlet three-brane on $X$ to obtain a BPS state whose mass is bounded by $\left|\int_{X} \Omega\right|$, which is just the size of the 3 -cycle $X$. In particular, as $z \rightarrow 0$ one obtains a new massless charged particle, arising from the wrapped 3-brane! This explains a log singularity in the classical IIB prepotential. We will find even more dramatic examples of such phenomena in $\mathrm{N}=1$ compactifications, where nonperturbative dynamics of solitons will explain singularities of classical string theory.

We should note that a physical interpretation of the singular points is necessary for consistency of the theory. But more importantly, it allows one to gain control over novel physical effects. For instance:

- In $4 \mathrm{~d} \mathrm{~N}=2$ models, the quantum understanding of conifold points gives a physical realization to the idea of "conifold transitions." These allow one to connect up the moduli spaces of all CalabiYau compactifications of type II strings [2, 迎!

- In the $4 \mathrm{~d} N=1$ case, we will see later on that singular points allow one to connect up models with different net numbers of chiral generations 25)!

There is yet another motivation for studying singularities, which is generic to $\mathrm{N}=1$ supergravity models with superpotentials.

\subsection{Poles and Superpotentials}

It turns out that in $4 \mathrm{~d} \mathrm{~N}=1$ models, singular points are necessary in order to have a nonvanishing superpotential 26].

Recall that $\mathrm{N}=1$ supergravity theories are specified by a Kahler potential $K\left(\Phi, \Phi^{*}\right)$ as well as a holomorphic superpotential $W(\Phi)$ and gauge coupling function $f(\Phi)$. 
- The Lagrangian $\mathcal{L}$ has a Kahler invariance under which

$$
K\left(\Phi, \Phi^{*}\right) \rightarrow K+F(\Phi)+F^{*}\left(\Phi^{*}\right)
$$

This Kahler transformation must be accompanied by a chiral transformation on the fermion fields in $\Phi$ and the gravitino.

- In the presence of a superpotential $W$, the Kahler invariance still holds if in addition to the shift of $K$, one has

$$
W \rightarrow e^{-F} W
$$

This means that $\mathrm{W}$ is actually a section of a line bundle over the moduli space $\mathcal{M}$ parametrized by the $\Phi$ s.

- The connection on this bundle has covariant derivatives

$$
\begin{gathered}
\frac{D U}{D \phi}=\frac{\partial U}{\partial \phi}+\frac{\partial K}{\partial \phi} U \\
\frac{D U}{D \phi^{*}}=\frac{\partial U}{\partial \phi^{*}}
\end{gathered}
$$

from which it follows that its curvature is $-\frac{\partial^{2} K}{\partial \phi \partial \phi^{*}}=-g_{\phi \phi^{*}}$. Since to get the right sign of the kinetic energy we want the metric on $\mathcal{M}$ to be positive definite, this means that the superpotential is a section of a line bundle of negative curvature over $\mathcal{M}$.

But now a standard theorem of complex geometry tells us that if $\mathcal{M}$ is compact, or can be suitably compactified, then as a section of a line bundle of negative curvature $W$ must have poles if it does not identically vanish 26]. This means that

a) The known tree level superpotentials in string theory must have poles at codimension one. We must explain these poles physically.

b) If we want to find "non-perturbative" superpotentials which e.g. dynamically break supersymmetry or destabilize would-be supersymmetric ground states, they too must have poles. So it is sometimes easiest to see if such a superpotential is generated by going to the potential singular points in moduli space (which are often clearly identifiable in a given model) and checking there.

\section{The Simplest Singularities of Heterotic Strings on K3}

Our eventual goal is to study singularities of $4 \mathrm{~d} \mathrm{~N}=1$ compactifications of the heterotic string. However, our strategy will be to use knowledge of singular behavior in six dimensions, fibered over an additional sphere, to determine the fourdimensional physics. So we first need to review the phenomena which accompany singularities in $6 \mathrm{~d}(0,1)$ supersymmetric heterotic string compactifications. We will study the simplest cases here; more complicated singularities in $(0,1)$ vacua have recently been discussed in e.g. 27.

\subsection{Heterotic $\mathrm{SO}(32)$ on $\mathrm{K} 3$}

When we compactify the $S O(32)$ heterotic string on a $\mathrm{K} 3$ to obtain a $6 \mathrm{~d} N=1$ theory, the Bianchi identity

$$
d H=\operatorname{Tr}(R \wedge R)-\operatorname{Tr}(F \wedge F)
$$

together with the fact that $\int_{K 3} \operatorname{Tr}(R \wedge R)=24$ tells us that we must put 24 Yang-Mills instantons in the $S O(32)$. The natural place to look for singularities is then when one of the instanton scale sizes shrinks to zero. The novel physics of such points was explained by Witten 28].

What can possibly happen at such a point? Singular points in the moduli space are most simply explained as points where new particles are becoming light. In $6 \mathrm{~d}$ theories with $(0,1)$ supersymmetry, the only massive supermultiplet with spins $\leq 1$ is the massive vector multiplet. As its mass goes to zero, it becomes a massless vector + hypermultiplet. So the only relevant free low energy dynamics is that the Higgs mechanism might be turned on or off.

Consider a shrinking instanton in $S O(N)$ then. Since it is small the details of the $K 3$ are irrelevant, so we might as well pretend it is on $R^{4}$. Then

$$
\begin{gathered}
\operatorname{dim}\left(\mathcal{M}_{i n s t}\right)=4 N-8 \\
\mathcal{M}_{\text {inst }}=\mathcal{M}_{i n s t}^{\prime} \times R^{4} \\
\operatorname{dim}\left(\mathcal{M}_{\text {inst }}^{\prime}\right)=4 N-12
\end{gathered}
$$

where the $R^{4}$ factor is just the instanton center of mass. Now if one proposes to get $\mathcal{M}_{\text {inst }}^{\prime}$ by 
Higgsing a gauge group $G$ with $k$ hypermultiplets, then

$$
\operatorname{dim}\left(\mathcal{M}_{\text {inst }}^{\prime}\right)=4(k-\operatorname{dim}(G))
$$

So one needs $k-\operatorname{dim}(G)=N-3$. Hence, it is natural to conjecture that $G=S U(2)$ and the matter is $\mathrm{N}$ half-hypermultiplets in the $\mathbf{2}$ of $S U(2)$.

At least two strong pieces of evidence favor the conjecture that a nonperturbative $S U(2)$ together with an $(\mathbf{N}, \mathbf{2})$ of $S O(N) \times S U(2)$ halfhypermultiplets appear when an $S O(N)$ instanton shrinks to zero size.

1) There is a well known ADHM construction of instanton moduli space 29]. The moduli space of the proposed nonperturbative gauge theory precisely reproduces this construction.

2) There is a description of instantons at scale sizes $\rho>>l_{s}$ on $K 3$ as the $(0,4)$ superconformal field theory of "solitonic fivebranes" of thickness $\rho$. As $\rho \rightarrow 0$, the fivebrane goes to zero thickness. Since this whole process is independent of $g_{\text {string }}$, we can take $g_{\text {string }} \rightarrow \infty$. Then by heterotic/ type I duality one obtains a weakly coupled type I string. It is natural to think that the zero thickness object you have sitting at a point on $K 3$ is now nothing but a type I Dirichlet 5-brane! The properties of the D5 brane are in complete agreement with the previous conjecture.

One can similarly see, as is clear from 2) above, that shrinking $k$ instantons at the same (smooth) point yields an $S p(k)$ gauge theory.

\subsection{Singularities of Heterotic $E_{8} \times E_{8}$ on K3}

The $E_{8} \times E_{8}$ heterotic string on $K 3$ can be thought of as M theory on $S^{1} / Z_{2} \times K 3$ [30]. Naively, the Bianchi identity for the $H$ field tells one that one must place $n_{1,2}$ instantons in the two $E_{8} \mathrm{~s}$ at the ends of the world, where

$$
n_{1}+n_{2}=24
$$

If for simplicity we put the $n_{1,2}$ instantons in an $S U(2)$ subgroup of each $E_{8}$, then the unbroken gauge symmetry on each end of the world is $E_{7}$, and the charged matter consists of $\left|\frac{1}{2} n_{1,2}-2\right| \mathbf{5 6}$ s. One can now ask, what happens as one of these $E_{8}$ instantons shrinks to zero size?

In $\S 5.1$, we saw that the only possible free IR dynamics consistent with $(0,1)$ supersymmetry is that the Higgs mechanism can be turned on or off. This insight sufficed in determining the behavior of the $S O(32)$ small instanton. However, it is easy to convince oneself that the singularity in the $E_{8}$ case must involve more subtle behavior than this (a quick glance at the dimensions of $E_{8}$ representations makes this clear). In fact, as explained in 31,32], the six-dimensional theory at the end of the world is governed by a nontrivial fixed point as an instanton shrinks to zero size. This is a multicritical point. The zero-size instanton constitutes a fivebrane at the end of the world. There are two phases connected by the fixed point theory: the Higgs phase is obtained by enlarging the small instanton, while a distinct Coulomb-like phase is obtained by moving the fivebrane off into the eleven-dimensional bulk. The Bianchi identity is generalized to [33]

$$
n_{1}+n_{2}+n_{5}=24
$$

where $n_{5}$ is the number of fivebranes. It follows from the previous discussion that the number of $1 / 256$ s at the end of the world the fivebrane leaves decreases by one.

The fivebrane worldvolume theory contains a tensor multiplet consisting of a real scalar, an antisymmetric tensor with selfdual field strength, and fermions. The real scalar's VEV gives the distance of the fivebrane from the end of the world. The perturbative heterotic theory has one tensor multiplet, whose two-form potential combines with that in the $6 \mathrm{~d}$ gravity multiplet (which has anti self-dual field strength) to form an unconstrained antisymmetric tensor field. The transition back to the perturbative heterotic theory from the phase with extra tensor multiplets cannot be described in conventional Lagrangian field theory, since the extra tensors cannot pick up masses without having any anti self-dual partners to couple to. 


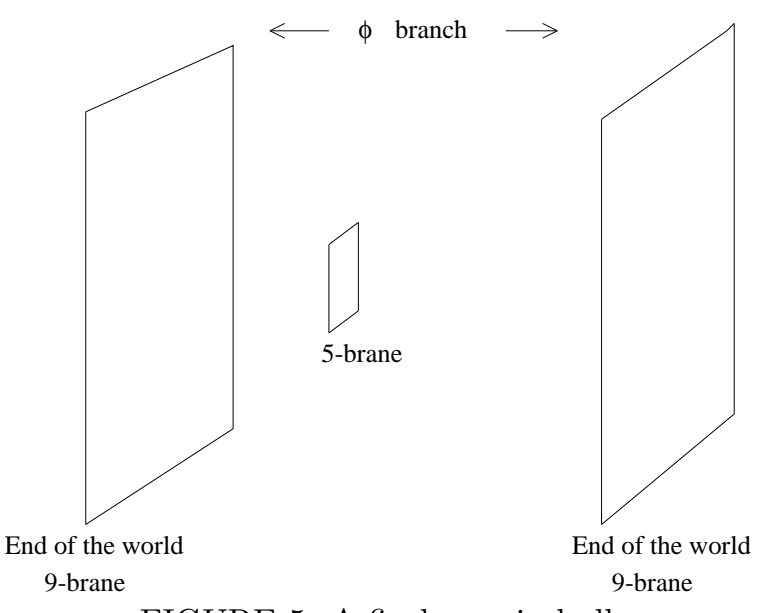

FIGURE 5: A fivebrane in bulk.

If we consider a single fivebrane and call the real scalar in the tensor multiplet $\phi$, then there are noncritical strings of tension $\phi$ and $\frac{1}{\alpha^{\prime}}-\phi$ arising from membranes stretched between the fivebrane and the two ends of the world. As the fivebrane approaches an end of the world, the tension of the associated noncritical string goes to zero. For this reason, the nontrivial fixed points which occur when an $E_{8}$ instanton shrinks to zero size are sometimes called "tensionless string theories." These fixed points (and their close relatives, obtained by compactifying on circles or tori) have been extensively investigated, in e.g. 34.

\section{K3 Fibrations and $S O(32)$ Strings}

So far, we have studied the simplest singularities of the heterotic theories compactified on K3 to six dimensions. Our real interest is to use this knowledge to understand singularities in the $4 \mathrm{~d}$ $\mathrm{N}=1$ theories coming from compactifications on Calabi-Yau threefolds which are K3 fibrations. If we study singularities which occur at a point in the generic K3 fiber, then we should be able to use our knowledge of the six-dimensional physics to learn about the four-dimensional physics. We proceed to do this for the $S O(32)$ string in this section, and discuss the $E_{8} \times E_{8}$ string in $\S 7$.

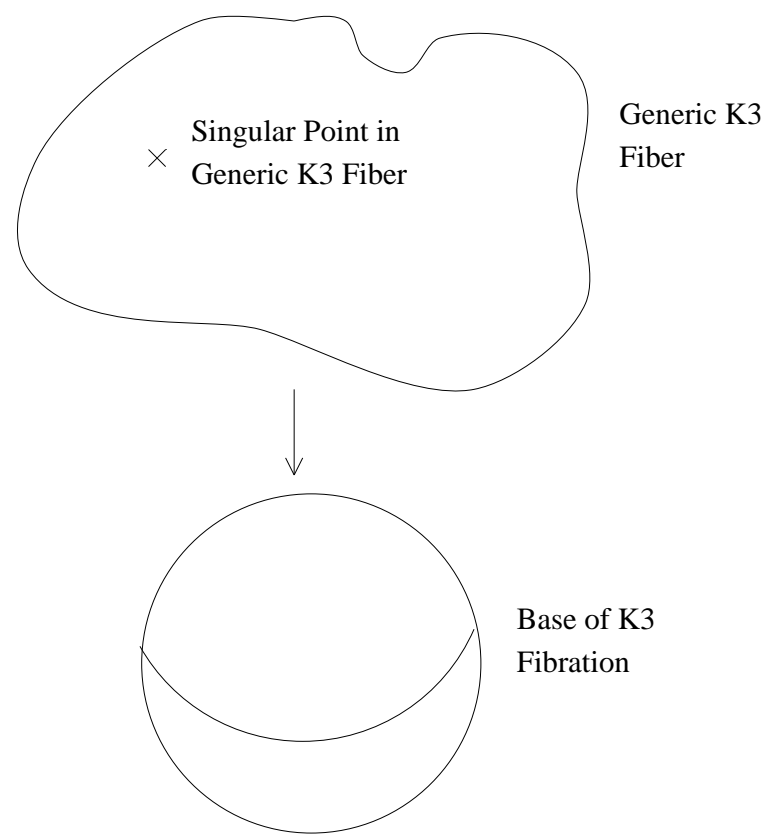

FIGURE 6: A K3 fibration with singularity in the generic fiber.

The simplest situation which we are equipped to study is the case that the K3 fibration $M$ has a single small instanton in the generic fiber. Then the generic fiber theory has an enhanced nonperturbative $S U(2)$ symmetry as described in $\S 5.1$. We will now see how this "fibers" to yield the four-dimensional story.

\subsection{Finding the $4 \mathrm{~d}$ Gauge Theory}

The question we need to answer is: What of the $S U(2)$ gauge theory with $321 / 2$ 2s of hypermultiplets survives in the $4 \mathrm{~d} N=1$ theory? The method we will use is to analyze adiabatically by making the $P^{1}$ base $C$ large and compactifying the $6 \mathrm{~d}$ theory on $C$ with a twist, to preserve precisely $4 \mathrm{~d} N=1$ supersymmetry [35.

The Lorentz group in the "K3 part" of the theory decomposes as $S O(4) \rightarrow S U(2)_{H} \times S U(2)^{\prime}$, where $S U(2)_{H}$ is the holonomy group of K3. The six dimensional supercharges are in a doublet of $S U(2)^{\prime}$, with $J_{3}^{\prime}= \pm 1 / 2$. The Lorentz group of $C$ is a $U(1)$, and each $6 \mathrm{~d}$ supercharge decomposes into pieces with $q= \pm 1 / 2$ under this $U(1)$. So if 
we twist and call the Lorentz $U(1) q+J_{3}^{\prime}$, we will end up keeping precisely half of the six dimensional supersymmetry.

The four-dimensional $S U(2)$ gauge bosons also survive the reduction as constants on $C$. So we will still get a nonperturbative $S U(2)$ gauge theory. The real question is, how many doublets survive?

For the gauge bundle $V \rightarrow M$ an $S U(N)$ bundle, the $(\mathbf{3 2}, \mathbf{2})$ splits into $2 \mathrm{~N}$ fields which couple to $V$ or $V^{*}$ and $32-2 \mathrm{~N}$ which couple to the trivial bundle. To count how many fermions survive (the bosonic spectrum follows by SUSY), we just need to count the number of zero modes of the Dirac operator on $C$. This counting is possible using several simple observations:

- Zero modes of $\not \partial+i A$ on $C$, where $\not \partial$ is the covariant Dirac operator and $A$ is the gauge connection on $V$, are in one to one correspondence with elements of $H^{0}(\mathcal{O}(-1) \otimes V)$. Here $\mathcal{O}(n)$ simply denotes the line bundle on $C$ with first Chern class $n$, and $\mathcal{O}(-1)$ is the spin bundle.

- It is also true that any holomorphic bundle $V$ splits as a direct sum of line bundles when it is restricted to $C$

$$
\left.V\right|_{C}=\sum_{i=1}^{N} \mathcal{O}\left(a_{i}\right)
$$

- Finally, to count the surviving fermions one needs to know that $h^{0}(\mathcal{O}(n))=n+1$ for $n \geq 0$ and vanishes for $n<0$.

Putting these facts together, we find that the $32-2 \mathrm{~N} 2 \mathrm{~s}$ which couple to the trivial bundle do NOT survive, since $H^{0}(\mathcal{O}(-1))$ is trivial. On the other hand, the $2 \mathrm{~N}$ doublets which couple to $V$ and $V^{*}$ contribute

$$
\begin{gathered}
\text { \# 4d doublets }=h^{0}(V \otimes \mathcal{O}(-1)) \\
+h^{0}\left(V^{*} \otimes \mathcal{O}(-1)\right)
\end{gathered}
$$

So on curves of singularities of the sort I have been describing, there is a nonperturbative enhancement of the spectrum consisting of $S U(2)$ gauge fields with a calculable number of doublets. The dynamics in this extra sector should allow us to explain various singularities of $S O(32)$ string compactifications.
Note that the coupling of the $S U(2)$ is not the normal string coupling of the heterotic string. Instead, it is

$$
\begin{gathered}
\frac{1}{g^{2}}=\frac{R^{2}}{\alpha^{\prime}} \rightarrow \\
\Lambda_{S U(2)} \sim M_{S} e^{-\frac{R^{2}}{\alpha^{\prime}}}
\end{gathered}
$$

where $R$ is the radius of the base curve $C$. On the other hand, in the type I theory these gauge fields are governed by the string coupling too.

\subsection{Uses of the $4 d$ Spectrum}

There are two phenomena which occur in heterotic $(0,2)$ models which can be explained by these nonperturbative $S U(2)$ gauge theories.

1) Worldsheet instanton destabilization

There can exist curves $C$ with worldsheet instantons (nontrivial maps of Euclidean string worldsheets which wrap $C$ ) contributing

$$
W \sim e^{-\frac{\int_{C} J}{\alpha^{\prime}}} \sim e^{-\frac{R^{2}}{\alpha^{\prime}}}
$$

to the spacetime superpotential [10]. It is of interest to know for which models such a superpotential appears, and to give an "explanation" of the superpotential in terms of conventional lowenergy physics.

2) Poles in Yukawa couplings

If we compactify the $S O(32)$ heterotic string on a K3 fibration $M$ and take $V \rightarrow M$ to be a holomorphic deformation of $T M$, then the low energy effective theory has $S O(26) \times U(1)$ gauge group. The charged matter content consists of

$$
\begin{aligned}
& h^{1,1}(M) \times 26_{1} \oplus 1_{-2} \\
& h^{2,1}(M) \times 26_{-1} \oplus 1_{2}
\end{aligned}
$$

Quantities of interest in $\mathcal{L}_{\text {eff }}$ include the Yukawa couplings

$$
y_{i j k} 26_{1}^{i} 26_{1}^{j} 1_{-2}^{k}
$$

where $y_{i j k}$ is a function of the moduli.

It has been observed that in many models 22, 11] the following occurs: If $z$ is a modulus of the theory such that at $z \rightarrow 0 V \rightarrow M$ becomes singular, then $y_{i j k}$ behaves like

$$
y_{i j k} \sim \frac{1}{z} \tilde{y}_{i j k}
$$


as $z \rightarrow 0$, where $\tilde{y}_{i j k}$ is finite in the limit. It is necessary for consistency of the theory to find a dynamical explanation for such poles.

We will try to explain phenomena 1) and 2) in turn, for this class of singularities.

\section{Application I: Vacuum Cleaning}

Suppose that at codimension one in the moduli space of vacua, one finds a singularity of the sort we've been discussing with

$$
\left.V\right|_{C}=\mathcal{O}(1) \oplus \mathcal{O}(-1) \oplus \mathcal{O}(0) \oplus \cdots
$$

where $\cdots$ denotes further trivial factors. Then one gets a nonperturbative enhancement of the spectrum consisting of $S U(2)$ gauge theory with 2 massless doublets $d_{1,2}$.

- Classically, this theory has a 1 complex dimensional moduli space of vacua with $V_{12}=\epsilon_{\alpha \beta} d_{1}^{\alpha} d_{2}^{\beta}$ being the (gauge invariant) coordinate.

- Quantum mechanically, Affleck, Dine and Seiberg showed some years ago [36] that a one instanton effect in this theory dynamically generates a superpotential

$$
W_{d y n}=\frac{\Lambda^{5}}{V_{12}}
$$

where for us $\Lambda \sim M_{S} e^{-\frac{R^{2}}{\alpha^{\prime}}}$. That is exactly the sort of superpotential we expect worldsheet instantons to generate in heterotic string theory! There are several checks:

i) The pole at $V_{12}=0$ can play the role of the pole required (as reviewed in $\S 4.2$ ) for a nonzero superpotential in supergravity.

ii) The fact that $C$ is a curve of singularities agrees perfectly with independent analysis of when a worldsheet instanton superpotential can be generated [11].

iii) By zero-mode counting arguments in an explicit worldsheet instanton computation, one can show [37] that this splitting type is one of only two cases where a worldsheet instanton superpotential is possible. This involves a counting of heterotic string worldsheet fermion zero modes instead of nonperturbative $S U(2)$ doublets!

The other splitting type consistent with a worldsheet instanton generated superpotential is trivial splitting. From our analysis, this case corresponds to a pure $S U(2)$ gauge theory, and gaug- ino condensation occurs, which again would generate a potential $W \sim \Lambda^{3}$ in the gauge theory analysis. In this case, since there are no moduli which remove the $S U(2)$, the compactification would be generically singular, however.

So, we have found that there is a complete agreement between situations where the nonperturbative $S U(2)$ gauge theory can lead to vacuum destabilization and situations where worldsheet instanton considerations allow a superpotential to be generated [38]. Three comments are in order: 1) Duality maps these worldsheet instanton effects in the heterotic string to both spacetime instantons of the type I theory (in the worldvolume gauge theory on a 5-brane), and Euclidean 5-branes wrapping divisors of arithmetic genus 1 in F-theory on a dual Calabi-Yau fourfold [39].

2) The physical upshot is that many heterotic or type I vacua with no nontrivial dynamics in the obvious (perturbative) gauge groups can still be destabilized by dynamics in less obvious nonperturbative gauge groups.

3) The techniques of [39 have been used to study considerably more elaborate examples of nonperturbative superpotentials, which exhibit modular behavior 40,41.

Application II: Poles in Yukawa Couplings

Analyzing the situations where the generic $K 3$ fiber is singular and there is a pole in a Yukawa coupling, we find that

$$
\left.V\right|_{C}=\mathcal{O}(2) \oplus \mathcal{O}(0) \oplus \mathcal{O}(-2)
$$

This implies by our previous analysis that at the singular point in moduli space one finds an $S U(2)$ gauge theory with $N_{F}=2$, i.e. 4 doublets.

So how does this explain the pole in the Yukawa couplings of $S O(26) \times U(1)$ charged matter [35]? Denote the doublets $d_{i}, 1 \leq i \leq 4$. As we reviewed in $\S 4.1$, SUSY QCD with $N_{F}=N_{C}=2$ has a smooth quantum moduli space $\mathcal{M}_{q u}$ which is a deformation of the classical one:

$$
\mathcal{M}_{q u}: \operatorname{Pf}(V)=\Lambda^{4}
$$

where $\Lambda$ is the dynamical scale of the gauge theory. The constraint restricting the fields to lie on $\mathcal{M}_{q u}$ can be imposed with a Lagrange multiplier 
in the superpotential

$$
W_{0}=\lambda\left(P f(V)-\Lambda^{4}\right)
$$

In the gauge theory, the Higgs branch has 5 complex dimensions. In our problem, there is only one relevant dimension in the moduli space, parametrized by $z$. This implies the existence of a tree-level superpotential

$$
\delta W=V_{13}^{2}+V_{14}^{2}+V_{23}^{2}+V_{24}^{2}
$$

Integrating out the $V$ s appearing in $\delta W$, we find that they vanish. Then the Pfaffian constraint obtained by integrating out $\lambda$ reveals

$$
V_{34}=\frac{\Lambda^{4}}{V_{12}}
$$

This yields the required complex 1-dimensional moduli space.

What about the pole? Consider adding a term

$$
\delta \tilde{W}=\left(26_{1} 26_{1} 1_{-2}\right) V_{34}
$$

to the superpotential. Then altogether we have

$$
W=W_{0}+\delta W+\delta \tilde{W}
$$

Now integrating out $V_{13}, V_{14}, V_{23}, V_{24}$ and $\lambda$, we find

$$
W_{d y n}=\frac{\Lambda^{4}}{V_{12}}\left(26_{1} 26_{1} 1_{-2}\right)
$$

This is the desired pole, with $z \sim V_{12}$ !

Note that nonperturbative quantum phenomena in the $S U(2)$ gauge theory can explain classical heterotic string singularities because $e^{-\frac{R^{2}}{\alpha^{\prime}}}$ is playing the role of $e^{-\frac{1}{h g^{2}}}$. This is the analogue of the special properties of RR fields used by Strominger in explaining classical singularities of $\mathrm{N}=2$ string vacua with quantum loops of light solitons [3].

\section{K3 Fibrations and $E_{8} \times E_{8}$ Strings}

We will now briefly discuss a similar analysis of singular $E_{8} \times E_{8}$ string compactifications on $K 3$ fibrations. This will reveal a novel phenomenon which we did not encounter in the $S O(32)$ case. We will basically motivate and summarize the results of [25], where all of the details can be found.
One of the longest standing mysteries of string theory has been the vacuum degeneracy problem. It is hard to see how a dynamical selection principle could choose between disconnected components of the space of vacua. For the case of compactifications with $\mathrm{N}=2$ supersymmetry, the situation improved considerably with the work of 幻.

The case of $\mathrm{N}=1$ vacua is qualitatively different from the $\mathrm{N}=2$ case. $\mathrm{N}=1$ is the first case where chiral gauge representations occur. No transition which occurs in weakly coupled Lagrangian field theory can change the net number of chiral generations. Nonetheless, we will find in this section that in fact some vacua of the $E_{8} \times E_{8}$ string with different generation numbers can be connected by phase transitions [25]. These transitions involve going through a point in moduli space where there is no free long distance description of the physics.

\subsection{Fibration Picture of the Phase Tran- sition}

As in $\S 6$, we consider compactification on a K3 fibration $M$ with base sphere $C$. Call the radius of $C R$. Above the scale $1 / R$, the physics can be analyzed in terms of an effective six-dimensional compactification on $K 3$.

Suppose an $E_{8}$ small instanton develops in the generic fiber. For simplicity, suppose it develops in an $E_{8}$ factor which is on a locus with unbroken $E_{7}$. Then as we discussed in $\S 5.2$, moving the small instanton fivebrane off into the bulk (i.e., turning on the scalar in its tensor multiplet) yields a vacuum with one less $1 / 2 \mathbf{5 6}$ localized on the wall it left. At the intersection of the two branches, the physics is governed by a nontrivial RG fixed point.

Let us now consider the theory below the scale $1 / R$, i.e. in four dimensions. For definiteness let us consider an $S U(3)$ bundle $V$ embedded in one $E_{8}$ on the Calabi-Yau threefold $M$. This leads to an $E_{6}$ gauge symmetry in spacetime. In a class of examples, an analysis of the $4 \mathrm{~d}$ spectrum reveals the following [25]: Each $6 \mathrm{~d} 1 / 2 \mathbf{5 6}$ hypermultiplet descends to a set of $\mathbf{2 7 \mathrm { s }}$ or to a $\overline{\mathbf{2 7}}$ in the $4 \mathrm{~d}$ theory. There is also some matter which does not descend from matter in the $6 \mathrm{~d}$ theory, i.e. which is not associated with the generic fiber. This matter 
is therefore associated with singular fibers.

In the $4 \mathrm{~d}$ theory, a zero-size instanton in the generic fiber corresponds to a fivebrane wrapped around the base $P^{1}$. As shown in [42] it remains consistent with supersymmetry for the fivebrane to move away from the end of the world, as long as it is wrapped on a holomorphic curve. In this phase, since of the the $1 / 256 \mathrm{~s}$ has been removed in six dimensions, the corresponding $\mathbf{2 7}$ s or $\overline{\mathbf{2 7}}$ have been removed in the four-dimensional theory. One can argue that no additional states from singular fibers are produced in the transition. So we find that the net generation number has shifted. This is possible because at the point of transition, there is no weakly coupled description of the infrared physics: The theory is governed by a nontrivial RG fixed point obtained by "fibering" the $6 \mathrm{~d}$ fixed point over a sphere. It would be very interesting to obtain a better understanding of such exotic $4 \mathrm{~d} \mathrm{~N}=1$ fixed points; examples have been constructed on brane probes in $[43]$.

\subsection{Instanton Effects}

So far, we have discussed the $4 \mathrm{~d}$ theory below the scale $1 / R$ set by the Kaluza-Klein modes on the sphere. There are also lower dynamical scales in the problem, set by the strength of worldsheet instanton effects. As we mentioned in $§ 5.2$, in addition to the fundamental string there are two noncritical strings of interest when the fivebrane moves off into the interval. If $\phi$ parametrizes its position on $S^{1} / Z_{2}$, then the tension of these noncritical strings goes like $\phi$ and $\frac{1}{\alpha^{\prime}}-\phi$. So euclidean worldsheets of these strings wrapping $C$ will generate effects that go like $e^{-R^{2} \phi}$ and $e^{-R^{2}\left(\frac{1}{\alpha^{\prime}}-\phi\right)}$.

The physics of these instanton effects can be classified according to the splitting type of $V$ over $C$ at the small instanton singularity, as in $\S 6$. Actually, in general there is another vector bundle $V_{2}$ embedded in the second $E_{8}$. At the point where the fivebrane is "absorbed" into the second $E_{8}, V_{2}$ is also singular over $C$ and its splitting type is of interest. There are a few different cases one can consider, and we mention two of the simplest below. Note that T-duality relates this theory, compactified on an additional circle, to the $S O(32)$ theories we discussed in the pre- vious section. So in many cases, results about three-dimensional $S U(2)$ gauge dynamics are (indirectly) related to the phenomena under study here.

Case I: If $h^{0}\left(\left.V\right|_{C} \otimes \mathcal{O}(-1)\right)$ and $h^{0}\left(\left.V_{2}\right|_{C} \otimes\right.$ $\mathcal{O}(-1))$ are both greater than two, then there is no worldsheet instanton generated superpotential on the $\phi$ branch. This follows from a standard counting of worldsheet instanton zero modes [37], which are associated to these two cohomology groups for the two kinds of noncritical strings. For these splitting types, there are too many zero modes for a potential to be generated. Furthermore, in these cases fundamental string worldsheet instanton effects also cannot remove the "small instanton" point in the classical moduli space, where the nontrivial fixed point theory occurs. The physics as analyzed at the scale $1 / R$ persists in the full quantum theory. Such vacua provide examples where the chirality change can occur while remaining in the moduli space of vacua, i.e. at zero cost in energy.

Case II: If $h^{0}\left(\left.V\right|_{C} \otimes \mathcal{O}(-1)\right)$ or $h^{0}\left(\left.V_{2}\right|_{C} \otimes\right.$ $\mathcal{O}(-1))$ is zero, a nonperturbative worldsheet instanton superpotential destabilizes the $\phi$ branch where the fivebrane is on the interval. One can see this in the $E_{8}$ theory by considering tensionful string worldsheet instantons of the first or second $E_{8}$. The number of zero modes on the worldsheet of such a string, for these splitting types, will be small enough for a nontrivial instanton-generated superpotential. This is consistent with known results about $S U(2)$ gauge theories in three dimensions 44, 45]. In this case, the chirality change which seems possible between the scale $\mu$ generated by the instanton superpotential and $1 / R$ is obstructed by quantum effects below $\mu$. However, the potential barrier which prevents chirality change in such cases can be hierarchically smaller than the string scale. This indicates that in these cases chirality change can "almost" occur, i.e. it is possible at a small cost in energy.

In closing: The insights from string duality have already been used to great effect in "solving" vacua with extended supersymmetry. In $4 \mathrm{~d}$ $\mathrm{N}=1$ compactifications, there has been progress in understanding methods of supersymmetry breaking and in removing an obstruction to unify- 
ing vacua. New techniques (particularly from F-theory/heterotic duality) are being developed [46, 47], and one should expect further progress in the near future.

\section{Acknowledgements}

Much of the work reported on in these notes was done in collaboration with E. Silverstein. I would also like to thank O. Aharony, P. Aspinwall, T. Banks, J. Distler, J. Louis, and N. Seiberg for discussions on some of these topics.

\section{REFERENCES}

1. J. Polchinski, Rev. Mod. Phys. 68 (1996) 1245, hep-th/9607050;

J. Schwarz, hep-th/9607201;

P. Aspinwall, hep-th/9611137;

C. Vafa, hep-th/9702201.

2. P. Green and T. Hubsch, Phys. Rev. Lett. 61 (1988) 1163;

P. Candelas, P. Green, and T. Hubsch, Phys. Rev. Lett. 62 (1989) 1956.

3. A. Strominger, Nucl. Phys. B451 (1995) 96, hep-th/9504090.

4. B. Greene, D. Morrison, and A. Strominger, Nucl. Phys. B451 (1995) 109, hepth/9504145;

T. Chiang, B. Greene, M. Gross, and Y. Kanter, hep-th/9511204;

A. Avram, P. Candelas, D. Jancic, and M. Mandelberg, Nucl. Phys. B465 (1996) 458, hep-th/9511230.

5. S. Kachru and C. Vafa, Nucl. Phys. B450 (1995) 69, hep-th/9505105.

6. S. Ferrara, J. Harvey, A. Strominger, and C. Vafa, Phys. Lett. 361B (1995) 59, hepth/9505162.

7. P. Candelas, G. Horowitz, A. Strominger and E. Witten, Nucl. Phys. B258 (1985) 46.

8. K. Uhlenbeck and S.T. Yau, Comm. Pure. App. Math. Vol XXXIX (1986) S257.

9. T. Hubsch, Calabi-Yau Manifolds, World Scientific, 1992.

10. M. Dine, N. Seiberg, X.G. Wen, and E. Witten, Nucl. Phys. B278 (1986) 769, Nucl. Phys. B289 (1987) 319.
11. E. Silverstein and E. Witten, Nucl. Phys. B444 (1995) 161, hep-th/9503212.

12. J. Polchinski and E. Witten, Nucl. Phys. B460 (1996) 525, hep-th/9510169.

13. M. Douglas, hep-th/9512077.

14. See e.g. B.S. Acharya, Nucl. Phys. B475 (1996) 579, hep-th/9603033 and references therein.

15. C. Vafa, Nucl. Phys. B469 (1996) 403, hepth/9602022.

16. C. Vafa and E. Witten, Nucl. Phys. B447 (1995) 261, hep-th/9505053.

17. S. Sethi, C. Vafa and E. Witten, Nucl. Phys. B480 (1996) 213, hep-th/9606122.

18. E. Witten, Nucl. Phys. B403 (1993) 159, hepth/9301042.

19. J. Distler and S. Kachru, Nucl. Phys. B413 (1994) 213, hep-th/9309110.

20. J. Distler and S. Kachru, Nucl. Phys. B442 (1995) 64, hep-th/9501111.

21. T. Chiang, J. Distler, and B. Greene, hepth/9702030.

22. P. Candelas, X. De la Ossa, P. Green, and L. Parkes, Nucl. Phys. B359 (1991) 21.

23. N. Seiberg, Phys. Rev. D49 (1994) 6857, hepth/9402044.

24. N. Seiberg and E. Witten, Nucl. Phys. B426 (1994) 19, hep-th/9407087.

25. S. Kachru and E. Silverstein, hep-th/9704185.

26. E. Witten and J. Bagger, Phys. Lett. 115B (1982) 202.

27. J. Blum and K. Intriligator, hep-th/9705044; P. Aspinwall and D. Morrison, hep-th/9705104, and references therein.

28. E. Witten, Nucl. Phys. B460 (1996) 541, hepth/9511030

29. M. Atiyah, N. Hitchin, V. Drinfeld, and Y. Manin, Phys. Lett. 65A (1978) 185.

30. P. Horava and E. Witten, Nucl. Phys. B460 (1996) 506, hep-th/9510209.

31. O. Ganor and A. Hanany, Nucl. Phys. B474 (1996) 122, hep-th/9602120.

32. N. Seiberg and E. Witten, Nucl. Phys. B471 (1996) 121, hep-th/9603003.

33. M. Duff, R. Minasian, and E. Witten, Nucl. Phys. B465 (1996) 413, hep-th/9601036.

34. N. Seiberg, Phys. Lett. B388 (1996) 753, hep- 
th/9608111;

D. Morrison and N. Seiberg, Nucl. Phys. B483 (1997) 229, hep-th/9609070;

M. Douglas, S. Katz, and C. Vafa, hepth/9609071:

O. Ganor, D. Morrison and N. Seiberg, Nucl. Phys. B487 (1997) 93, hep-th/9610251.

35. S. Kachru, N. Seiberg, and E. Silverstein, Nucl. Phys. B480 (1996) 170, hepth/9605036.

36. I. Affleck, M. Dine, and N. Seiberg, Nucl. Phys. B241 (1984) 493.

37. J. Distler, Phys. Lett. B188 (1987) 431.

38. S. Kachru and E. Silverstein, Nucl. Phys. B482 (1996) 92, hep-th/9608194.

39. E. Witten, Nucl. Phys. B474 (1996) 343, hepth/9604030.

40. R. Donagi, A. Grassi, and E. Witten, Mod. Phys. Lett. A11 (1996) 2199, hepth/9607091.

41. G. Curio and D. Lust, hep-th/9703007.

42. E. Witten, Nucl. Phys. B471 (1996) 135, hepth/9602070.

43. O. Aharony, S. Kachru, and E. Silverstein, Nucl. Phys. B488 (1997) 159, hepth/9610205.

44. O. Aharony, A. Hanany, K. Intriligator, N. Seiberg and M. Strassler, hep-th/9703110.

45. I. Affleck, J. Harvey and E. Witten, Nucl. Phys. B206 (1982) 413.

46. R. Friedman, J. Morgan, and E. Witten, hepth/9701162.

47. M. Bershadsky, A. Johansen, T. Pantev, and V. Sadov, hep-th/9701165. 